\title{
Path Optimization of Multi-source Signal Feature Factor Decomposition and Its Application
}

\author{
Liu Yang ${ }^{1, a}$, Hanxin Chen ${ }^{1, b^{*}}$, Wenjian Huang ${ }^{1, c}$, \\ Jinmin Huang ${ }^{1, \mathrm{~d}}$ and Chenghao $\mathrm{Cao}^{1, \mathrm{e}}$
}

\author{
${ }^{1}$ No. 206, Guanggu First Road, Jiangxia District, Wuhan, Hubei, 430205,P.R.China \\ awgdyangliu@sina.com, ${ }^{b^{*}}$ pg01074075@163.com, chwjhere@126.com, \\ d15207176266@163.com, ${ }^{e}$ holyqs@163.com
}

\section{Keywords: Feature Factor; Decomposition Path; Optimization Algorithm; Fault Diagnosis}

\begin{abstract}
The non-linear process mechanical state is characterized by multivariate, strong coupling, many vibration sources, large signal noise and various random factors. The mechanical structure generates corresponding nonlinear faults and multi-source characteristic signals under nonlinear excitation. Multi-source characteristic signal propagation is complex and varied, and it also has a strong nonlinear signal decomposition relationship. The intrinsic relationship of the directional path of the multi-source matrix signal feature and the overall consistency optimization is a brand-new study of multi-source dynamic feature signal identification. This project proposes a feature factor signal propagation path optimization algorithm based on nonlinear system identification theory. Under the guidance of matrix signal analysis theory, the vector analysis, spatial geometry, optimization theory, denoising principle and error theory are applied to analyze and discuss the intrinsic relationship of the optimal decomposition path of multi-source signal feature factors. It is established that the optimal nonlinear relationship between state variables, failure modes and feature signals in time, frequency and space. Then, the optimization of feature factor decomposition path is applied to feature extraction of ultrasonic fault detection signals.
\end{abstract}

\section{Introduction}

According to different types and characteristics of mechanical faults, scholars have put forward their own theories and methods in fault signal feature extraction, artificial intelligence fault identification and information fusion[1-3]. Li Qiang and Yang studied mechanical weak signal detection techniques[4,5]. Sanz et al. analyzed the characteristics of transient signals based on wavelet transforms[6,7]. Yu and Pan propose effective time-frequency feature analysis and artificial intelligence identification theory and method[8]. Unfortunately, some of the above applications are only aimed at fault diagnosis of mechanical equipment in smooth operation. In the industrial production line, the mechanical system is subjected to non-steady-state excitation to generate a nonlinear multi-failure mode. The fault features, such as time-frequency information, contain rich nonlinear state information and mechanical nonlinear multi-fault feature information so that it is necessary to carry out multi-source nonlinear dynamic feature signal extraction and identification[9]. The intrinsic relationship and overall consistency optimization of the directional path of the multi-source matrix signal feature factor are the key technologies and difficulties that must be solved for multi-source dynamic feature signal identification. It is a brand-new study. Especially with the rapid development of modern industry, the requirements for quality monitoring and fault diagnosis of production processes are getting higher and higher, and the demand for mechanical multi-failure mode diagnosis technology under nonlinear operating conditions is imminent.

\section{Optimization Algorithm of Multi-Source Signal Feature Factor Decomposition Path}

The nonlinear process mechanical state has the characteristics of multivariate, strong coupling, multiple vibration sources, large signal noise and various random factors. The mechanical structure generates nonlinear failure modes and nonlinear multi-source characteristic signals under nonlinear 
excitation. The signal propagation of source eigenfactor is complex and varied, and it also has a strong nonlinear signal decomposition relationship[10]. The intrinsic relationship of the directional path of the multi-source matrix signal feature and the overall consistency optimization is a new research of multi-source dynamic feature signal identification. The path direction relationship of multi-dimensional feature matrix signal decomposition in mathematics will not be a simple description, its establishment is more complex and difficult to derive. This project proposes a feature-based signal propagation path optimization algorithm based on nonlinear system identification theory.

This paper presents a novel nonlinear system identification method based on linear time domain model and frequency domain analysis. The relationship between the dynamic characteristics of the mechanical system and the frequency domain parameters in the nonlinear multi-fault mode is established. This relationship model can reveal the dynamic physical mechanism changes caused by changes of the mechanical structure. The mathematical model of the dynamic mechanism of the mechanical structure system can be determined through system input and output. This mathematical model uses the NARMAE (Nonlinear Auto-Regressive Moving Average with Exogenous input) theory to establish the time domain model, and establishes the dynamic performance relationship of the mechanical system structure between the input excitation signal and the output response signal. This method considers that any defect in the mechanical system structure represents that the dynamic characteristics of the structure are affected by an unknown input signal or an interference signal. The model parameter variable representing the fault of the mechanical structure is displayed in the frequency domain response function. The basic idea is that the dynamic process of the physical system can establish the quantitative relationship between the excitation input and the system response through the time domain model based on the NARMAX theory. The physical property variables of the mechanical structure system can not only be reflected in the time domain model but also be reflected by changes in the frequency response function.

The NARMAE model structure provides a single-input, single-output consistent representation of nonlinear dynamic system, using a nonlinear difference equation expressed as follows:

$$
y(t)=f\left(y(t-1), \ldots, y\left(t-n_{v}\right), u(t-1), \ldots, u\left(t-n_{u}\right), \varepsilon(t-1)\right)+\varepsilon(t)
$$

$\mathrm{y}(\mathrm{t}), \mathrm{u}(\mathrm{t})$, and $\varepsilon(\mathrm{t})$ represent system output, system input, and prediction error, respectively. $\mathrm{f}($.$) is an$ unknown nonlinear function that needs to be determined by input and output data. The non-linear estimated value of the function $f$ is realized by solving the function package of the effective dimension parameter vector $\theta$. The formula is as follows:

$$
y^{\prime}(t)=f^{\prime}\left(y(t-1), \ldots, y\left(t-n_{y}\right), u(t-1), \ldots, u\left(t-n_{u}\right), \varepsilon(t-1), \mathrm{K}, \varepsilon\left(t-n_{\varepsilon}\right), \theta\right)
$$

The prediction error is defined as:

$$
\varepsilon(t)=y\left(t \rightarrow{ }^{\prime} y(\right.
$$

Assuming a system inputs and outputs data, the linear system model can effectively implement frequency response function of the measurement system. The system frequency response is interpreted using a conversion function and defined as:

$$
H\left(e^{j w}\right)=\frac{\sum_{k=0}^{M-1} b(k) e^{-j w k}}{\sum_{I=0}^{N-1} a(I) e^{-j w k}}
$$

When the frequency response function is obtained, an automatic program can be determined that any changes in the system parameters are evaluated by comparing the frequency response function to the prophetic mode. Under the framework of frequency analysis of NARMAX model and frequency response function, fault diagnosis of a complex process industrial system can be achieved by analyzing a model of a recognized nonlinear system. The system response function uses a series of one-dimensional frequency functions to represent the system frequency characteristics. This method framework can be widely applied to fault diagnosis of industrial systems and can solve 
many problems, such as identifying nonlinear models from a detected system, accurately solving frequency response functions, extracting representative frequency domain features from frequency response functions, and implementing fault diagnosis using the extracted system frequency domain feature. This project has been explored in the above aspects, and the application of non-destructive testing methods for mechanical structures in the industrial process has been demonstrated as follows.

\section{Multi-Source Signal Feature Factor Decomposition Path Optimization in Ultrasonic Fault Detection}

Figure 1 shows the basic idea of the PEC signal detection crack test method. The oscilloscope is used to transmit the excitation signal to the PEC sensor and receive the system response signal from the PEC sensor. Figure 2 displays the basic flow of a nonlinear system identification method based on a linear time domain model and a frequency response function. The frequency response function of the time domain model can be used as a parameter of different crack lengths. Different values of the frequency response function of the time domain model can be used to determine the different crack sizes.

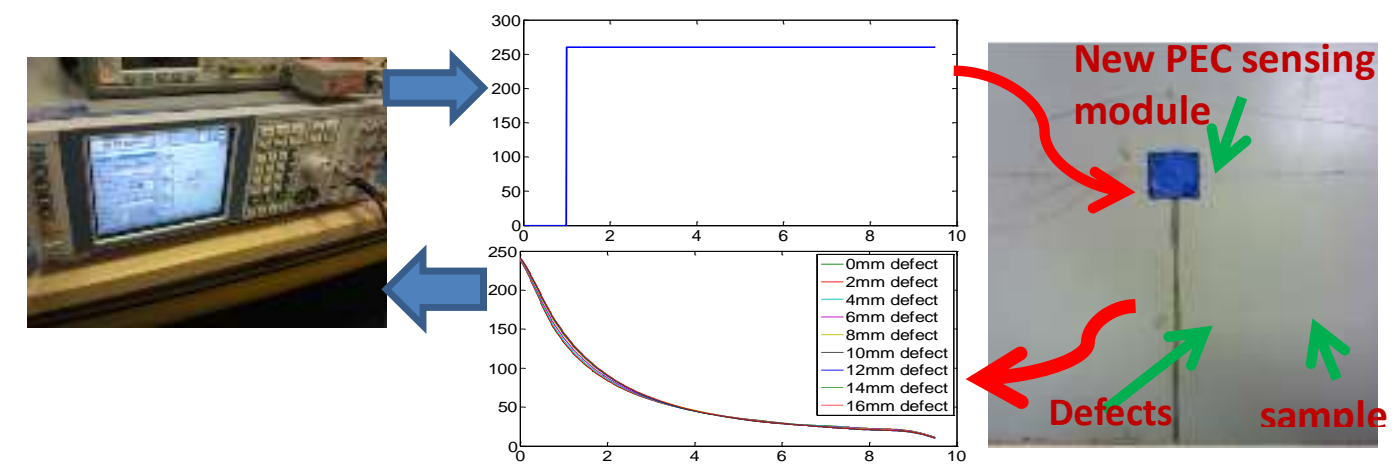

Figure 1. Material crack detection test system and data analysis 


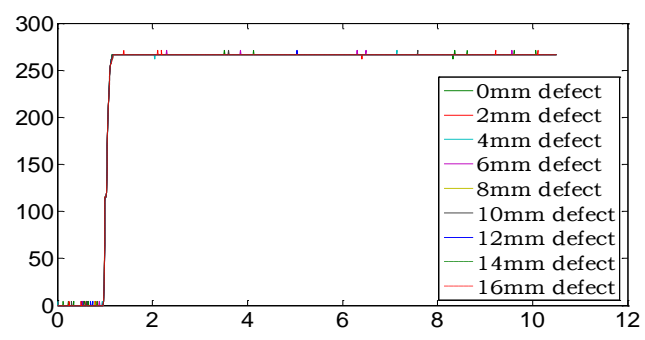

(a)Input signal

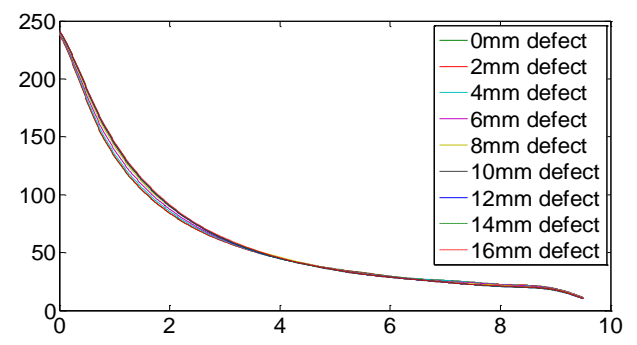

(b) Output signal

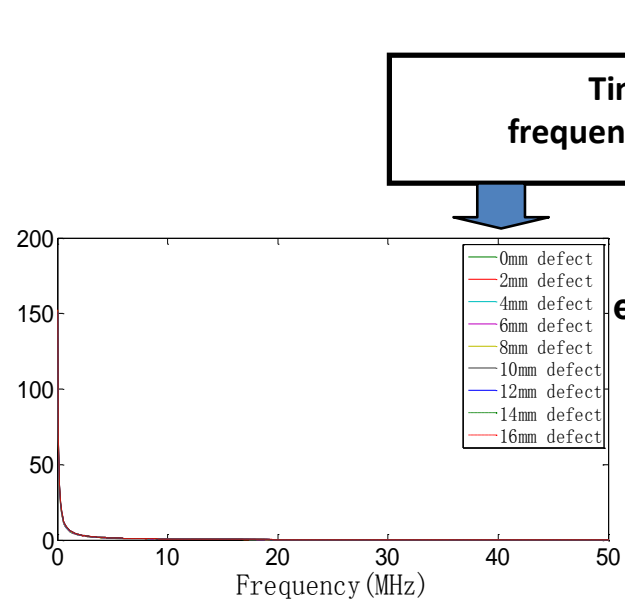

(c) System response
Time domain modeling and requency domain feature extraction

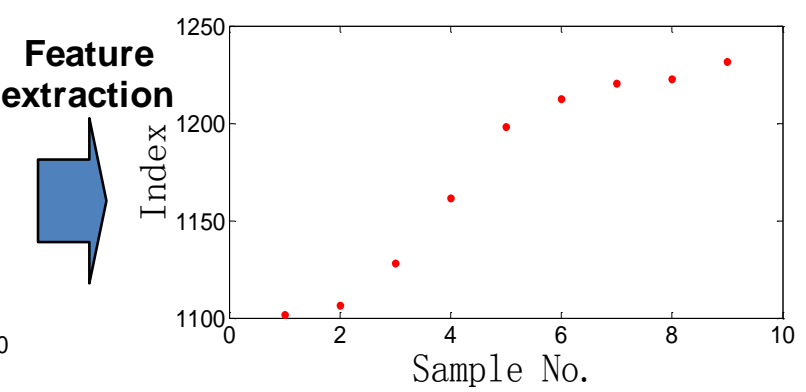

(d) Characteristic parameters

Figure 2. The basic flow of non-sexual system identification method based on linear time domain model and frequency response function

Figure 3 shows the idea of analyzing the corrosion of non-linear systems to detect materials. The frequency response function variables of the time domain model can identify the degree of material corrosion. The material corrosion wave is used to detect the corrosion of the material and the input signal is used to obtain the output signal. Based on the system response data, the time domain model is established and the frequency domain characteristic parameters are extracted. Finally, the corresponding corrosion crack parameters can be obtained so that the crack corrosion of the material is diagnosed. The above application examples show the effectiveness of the nonlinear system identification theory algorithm proposed in this project, which provides theoretical and experimental basis for further in-depth study of process industry fault diagnosis. 

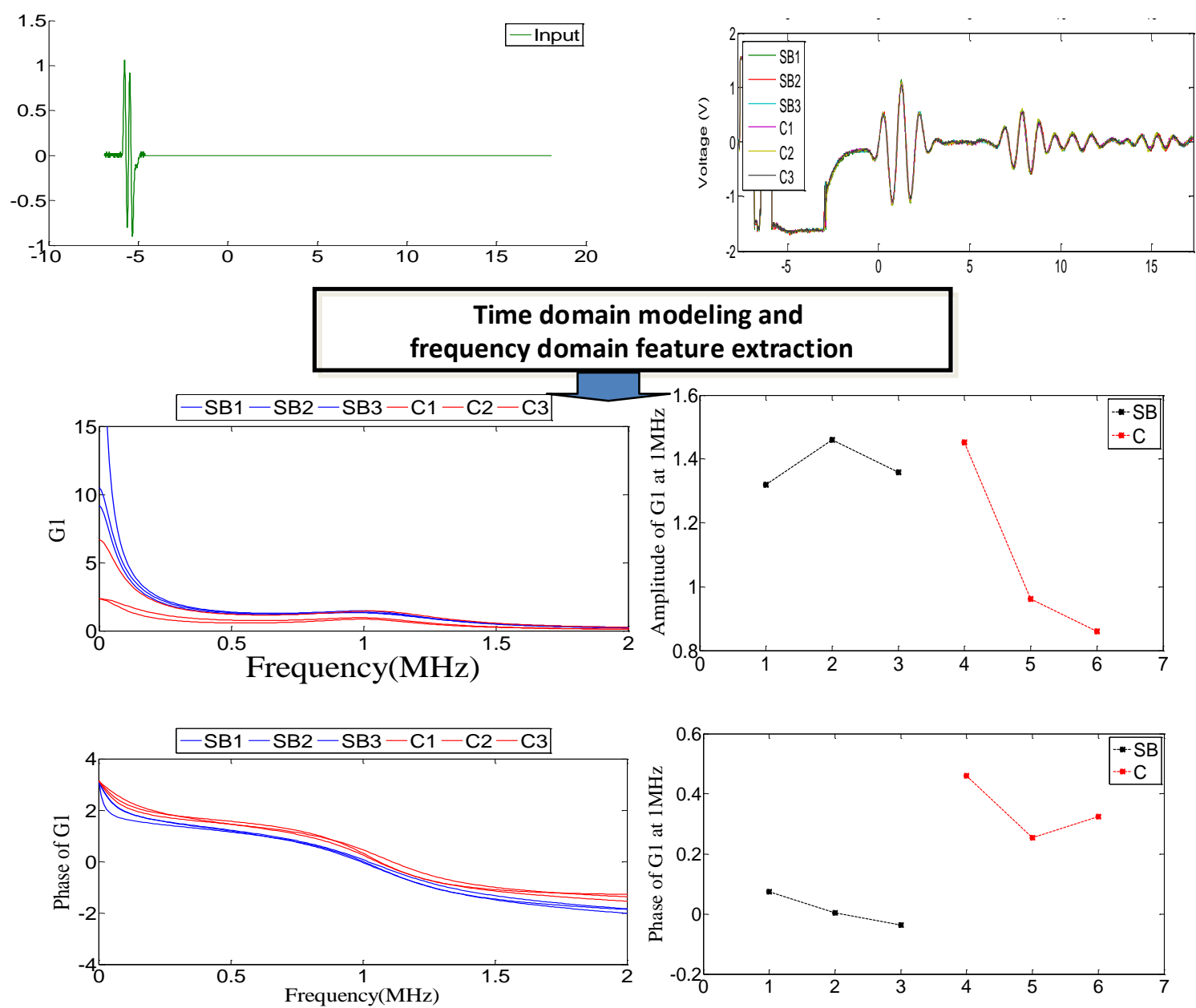

Figure 3. Frequency response function G1 amplitude characteristic parameter in material corrosion detection

\section{Conclusions}

This paper proposes a novel nonlinear system identification method based on linear time-domain model and frequency domain analysis and establishes a relation model between dynamic characteristics and frequency domain parameters of a mechanical system in a nonlinear multi-fault mode. This relationship model can reveal changes in the dynamic physical mechanism caused by changes in the mechanical structure. This model is applied to the material corrosion detection. The results show that the frequency response function variable of the time domain model can identify the degree of material corrosion in the nonlinear system identification and detection of material corrosion. This shows the effectiveness of the multi-source signal feature factor decomposition path optimization algorithm proposed in this project, which provides theoretical and experimental basis for further in-depth research in the process industry fault diagnosis.

\section{Acknowledgement}

This work was supported by the Special Major Project of theMinistry of Science and Technology of Hubei Province of China (Grant No.2016AAA056), Major project of Hubei Provincial Department of Education (Z20101501) and the National Natural Science Foundation of China (Grant No. 61273176,51775390). 


\section{References}

[1] Yu FS, Kang H, Zhang HW:Fault diagnosis for hydraulic drilling rig based on BP neural network optimized by PSO.Process Autom Instrument 37(4) (2016):42-56

[2] Banerjee TP, Das S: Multi-sensor data fusion using support vector machine for motor fault detection. Inf Sci 217(24) (2012):96-107.

[3] Gao SZ, Wang JS, Zhao N : Fault diagnosis method of polymerization Kettle equipment based on rough sets and BP neural network. Math Probl Eng 2013:1-8.

[4] Li Qiang, Wang Taiyong, Research on Weak Signal Detection Technology in Early Prediction of Mechanical Equipment, Tianjin University Doctoral Dissertation, 2008.

[5] Yang Jianwen, Jia Minping Zhong Binglin, The application of CSD analysis in the weak fault diagnosis of rotating machine, International Conference on Frontier of Design and Manufacturing (ICFDM'2006), Vol.2, 2006, pp.105-108.

[6] J.Sanz, R. Perara, and C. Huerta, Fault diagnosis of rotating machinery based on autoassociative neural networks and wavelet transforms, Journal of Sound and Vibration, Vol. 302, n4-5, 2007.

[7] Wang Qingjun, Hanxin Chen, Xubing Chen,Hong Yang and Gaoping Wang,Early fault detection of gearbox using weak vibration signals,Maintenance and Reliability,Jan,2011.

[8] Gao SZ,Wang JS and Zhao N.Fault diagnosis method of polymerization Kettle equipment based on rough sets and BP neural network.Math Probl Eng, 2013:1-8.

[9] Hanxin Chen,Yunfei Shang and Kui Sun,Sequential hypothesis testing for reflected signal recognition of time-of-flight estimation in ultrasonic defect detection,Insight-Non-destructive testing and condition monitoring,55(2),2013,1-6.

[10] Hanxin Chen, Ming J Zuo, Xiaodong Wang and Mohammad R Hoseini, An Adaptive Morlet WaveletFilter for Time-of-Flight Estimation in Ultrasonic Damage Assessment, Measurement, Vol.43, 2010,pp.570-585. 